\title{
On the speed of a planar random walk avoiding its past convex hull
}

\author{
Martin P.W. Zerner* \\ Department of Mathematics, Stanford University, Stanford, CA 94305, USA
}

Received 12 June 2003; accepted 11 August 2004

Available online 25 January 2005

\begin{abstract}
We consider a random walk in $\mathbb{R}^{2}$ which takes steps uniformly distributed on the unit circle centered around the walker's current position but avoids the convex hull of its past positions. This model has been introduced and studied by Angel, Benjamini and Virág. We show a large deviation estimate for the distance of the walker from the origin, which implies that the walker has positive lim inf speed.
\end{abstract}

(c) 2005 Elsevier SAS. All rights reserved.

\section{Résumé}

On considère une marche aléatoire sur $\mathbb{R}^{2}$ avec des pas distribués uniformément sur le cercle unité centré sur la position courante de la marche mais n'entrant pas dans l'adhérence convexe de ses positions précédentes. Ce modèle a été introduit et étudié par Angel, Benjamini et Virag. On démontre une estimée de grandes déviations pour la norme de la marche, qui implique que le limite inférieure de la vitesse de la marche est positive.

( 2005 Elsevier SAS. All rights reserved.

$M S C$ : primary $60 \mathrm{~K} 35$; secondary $60 \mathrm{G} 50,52 \mathrm{~A} 22$

Keywords: Convex hull; Large deviations; Law of large numbers; Random walk; Self avoiding; Speed

\section{Introduction}

Angel, Benjamini and Virág introduced and studied in [1] the following model of a random walk $\left(X_{n}\right)_{n \geqslant 0}$ in $\mathbb{R}^{2}$, which they called the rancher. The walker starts at the origin $X_{0}=0$. Suppose it has already taken $n$ steps $(n \geqslant 0)$ and is currently at $X_{n}$. Then its next position $X_{n+1}$ is uniformly distributed on the unit circle centered around $X_{n}$ but conditioned so that the straight line segment $X_{n}, X_{n+1}$ from $X_{n}$ to $X_{n+1}$ does not intersect the convex hull $K_{n}$ of the past positions $\left\{X_{0}, X_{1}, \ldots, X_{n}\right\}$, see Fig. 1 .

\footnotetext{
* Current address: Mathematisches Institut, Universität Tübingen, Auf de Morgenstelle 10, 72076 Tübingen, Germany.

E-mail address: martin.zerner@uni-tuebingen.de (M.P.W. Zerner).
} 


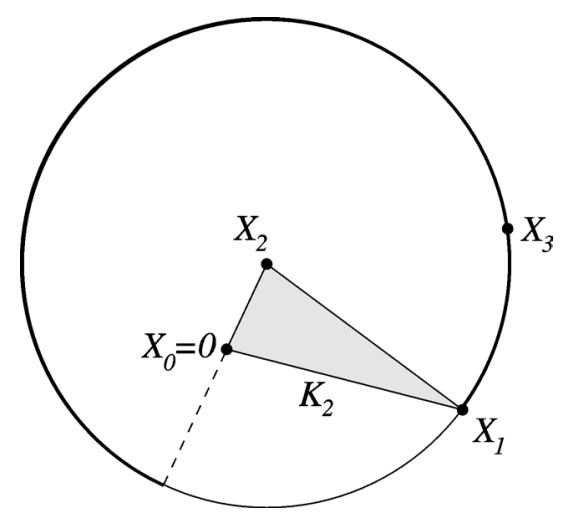

Fig. 1. Three steps of the walk. $X_{3}$ is uniformly distributed on the bold arc of the circle with radius 1, centered in $X_{2}$.

Note that $\left(X_{n}\right)_{n \geqslant 0}$ is not Markovian since in general one needs to know the whole history of the process in order to determine the transition probabilities for the next step. This makes this model more difficult to analyze than a Markovian random walk, a property it shares with many other self-interacting processes, see [1] and also [2] for references. We do not claim that this model is of particular importance or that its definition is very natural. However, in the class of all self-interacting processes it seems to be among the few models which are at least partially analyzable and still have some striking properties.

Several such properties have been conjectured by Angel, Benjamini and Virág in [1]. Based on simulations and heuristics, the authors of [1] believe e.g. that the direction of the walk converges, i.e. that $X_{n} /\left\|X_{n}\right\|$ converges $P$-a.s. to a random direction, see [1, Conjecture 1]. Moreover, if we denote by $w_{n}$ as a measure for transversal fluctuations the maximal distance of a point in $K_{n}$ from the line $\overline{X_{0}, X_{n}}$, then Angel, Benjamini and Virág conjecture that $w_{n}$ grows like $n^{3 / 4}$, see [1, Conjecture 2].

As far as results are concerned, the only major rigorous result which has been proved so far for this model to the best of our knowledge is that the walk has positive lim sup speed, i.e. there is a constant $c>0$ such that $P$-a.s. $\lim \sup \left\|X_{n}\right\| / n>c$ as $n \rightarrow \infty$, see [1, Theorem 1]. Here $(\Omega, \mathcal{F}, P)$ is the underlying probability space.

The purpose of the present paper is to improve this result by showing the following.

Theorem 1. There is a constant $c_{1}>0$ such that

$$
\limsup _{n \rightarrow \infty} \frac{1}{n} \log P\left[\left\|X_{n}\right\| \leqslant c_{1} n\right]<0
$$

and consequently,

$$
\liminf _{n \rightarrow \infty} \frac{\left\|X_{n}\right\|}{n} \geqslant c_{1} \quad P \text {-a.s. }
$$

In particular, (2) proves [1, Conjecture 4]. We expect but were not able to prove that the speed $\lim \left\|X_{n}\right\| / n$ exists and is $P$-a.s. constant, as conjectured in [1, Conjecture 5].

Let us now describe how the present article is organized. The next section introduces general notation and gives a short overview of the proof. In Section 3 we introduce some sub- and supermartingales, which enable us in Section 4 to bound exponential moments of the time it takes the diameter of the convex hull $K_{n}$ to increase. From this we deduce in Section 5 estimates for the diameter of $K_{n}$ similar to the ones claimed in Theorem 1 for $\left\|X_{n}\right\|$ and show how this implies Theorem 1. 


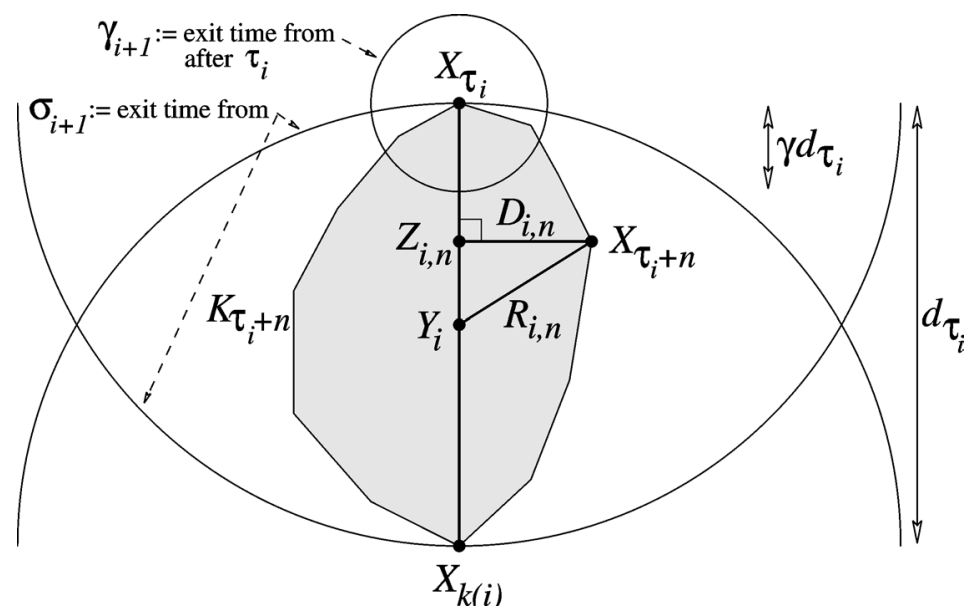

Fig. 2. General notation.

\section{Notation and outline of proof}

We denote by $d_{n}$ the diameter of $K_{n}$. Since $\left(K_{n}\right)_{n}$ is an increasing sequence of sets, $\left(d_{n}\right)_{n}$ is non-decreasing. The ladder times $\tau_{i}$ at which the process $\left(d_{n}\right)_{n \geqslant 0}$ strictly increases are defined recursively by

$$
\tau_{0}:=0 \quad \text { and } \quad \tau_{i+1}:=\inf \left\{n>\tau_{i}: d_{n}>d_{\tau_{i}}\right\} \quad(i \geqslant 0) \text {. }
$$

It follows from [1] that $P$-a.s. $\tau_{i}<\infty$ for all $i \geqslant 0$. In Section 4 we will reprove this fact by showing that the differences

$$
\Delta_{i}:=\tau_{i+1}-\tau_{i} \quad(i \geqslant 0),
$$

between two successive finite ladder times have some finite exponential moments. Note that $\tau_{1}=1$ since $d_{0}=0$ and $d_{1}=1$ and observe that the $\tau_{i}$ 's are stopping times with respect to the canonical filtration $\left(\mathcal{F}_{n}\right)_{n \geqslant 0}$ generated by $\left(X_{n}\right)_{n \geqslant 0}$. Since the diameter of a bounded convex set is the distance between two of its extremal points there is for all $i \geqslant 1$ with $\tau_{i}<\infty$ a $\left(P\right.$-a.s. unique) $0 \leqslant k(i)<\tau_{i}$ such that $d_{\tau_{i}}=\left\|X_{\tau_{i}}-X_{k(i)}\right\|$, see Fig. 2. For $x \in \mathbb{R}^{2}$ and $r>0$ we denote by $B(x, r)$ the closed disk with center $x$ and radius $r$. If $\tau_{i}<\infty$ then

$$
\sigma_{i+1}:=\inf \left\{n \geqslant 0 \mid X_{n} \notin B\left(X_{\tau_{i}}, d_{\tau_{i}}\right) \cap B\left(X_{k(i)}, d_{\tau_{i}}\right)\right\}
$$

is the exit time of the walk from the large lens shaped region shown in Fig. 2, which we shall refer to as the lens created at time $\tau_{i}$. Observe that $K_{\tau_{i}}$ is contained in the lens created at time $\tau_{i}$. Moreover,

$$
\tau_{i+1} \leqslant \sigma_{i+1}
$$

since if $\sigma_{i+1}<\infty, X_{\sigma_{i+1}}$ has a distance from either $X_{\tau_{i}}$ or $X_{k(i)}$ greater than $d_{\tau_{i}}$.

We have now introduced enough notation to be able to outline the idea of the proof of Theorem 1 .

Outline of Proof. To show that $\left(\left\|X_{n}\right\|\right)_{n}$ is growing at a positive rate we shall first show that $\left(d_{n}\right)_{n}$ is doing so. To this end we shall bound exponential moments of the $\Delta_{i}$ 's. This is the heart of the proof and done as follows.

Suppose that $d_{n}$ is already quite large and that we have just reached a new ladder time $\tau_{i}$, at which the diameter has increased. Due to (3) the diameter can stay constant only as long as the walk is hiding in the lens created at time $\tau_{i}$. However, there are two mechanisms, corresponding to Lemmas 4 and 5 , which ensure that the walk cannot hide in the lens for too long.

The first one, described in Lemma 4, works while the walk is still inside a small ball around $X_{\tau_{i}}$, see Fig. 2. The radius of this ball is chosen to be proportional to $d_{\tau_{i}}$. This situation is depicted in an idealized form in Fig. 3. In this 


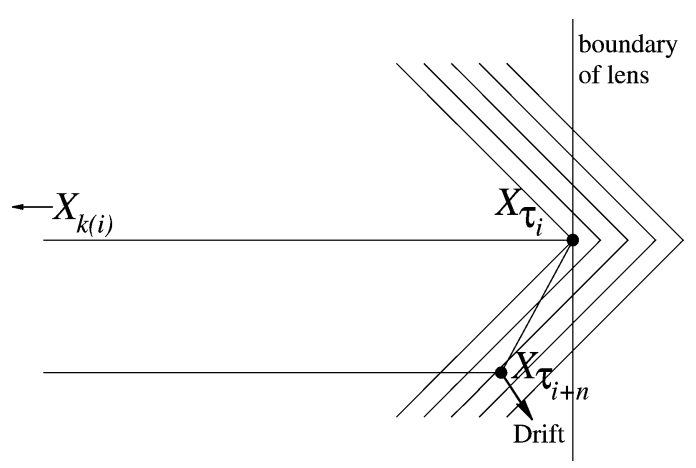

Fig. 3. Idealized picture, assuming $d_{\tau_{i}}=\infty$. Whenever the walker crosses a wedge for the first time, which occurs at a positive rate, it has in the next step a positive drift towards the boundary of the lens.

regime the following happens: Whenever the walk crosses for the first time any of the wedge-shaped lines shown in Fig. 3, it has a drift away from $X_{k(i)}$ towards the boundary of the lens. To see this consider the two boundary lines of the convex hull which are emanating from $X_{\tau_{i+n}}$ in Fig. 3. Suppose we are to draw in Fig. 3 the line going from $X_{\tau_{i+n}}$ to the left. In the picture this line has slope 0, i.e. it is parallel to $\overline{X_{\tau_{i}}, X_{k(i)}}$, but of course it could have a different slope. However, this slope cannot be much smaller than 0 because $X_{\tau_{i+n}}$ is close to $X_{\tau_{i}}$ but far away from $X_{k(i)}$. On the other hand, the line emanating from $X_{\tau_{i+n}}$ to the right must have in Fig. 3 a slope of at least 1. Together, these two lines create a drift downwards and more importantly to the right towards the boundary of the lens. (If the left line has a positive slope then the drift to the right is even stronger.)

So every time the walker crosses for the first time such a helpful wedge-shaped line it will be pushed towards the next wedge-shaped line. If the little ball around $X_{\tau_{i}}$ was infinitely large this would result in crossings of the wedgeshaped lines at a positive rate, which would propel the walk out of the lens very fast, i.e. this mechanism alone would give a finite exponential moment of $\Delta_{i}$, see Lemma 4. However, since the ball's size is finite and proportional to $d_{\tau_{i}}$, the walk can escape from this little ball before leaving the lense with a probability exponentially small in $d_{\tau_{i}}$. (This will happen a finite number of times.)

Whenever this occurs, we rely on the second mechanism, see Lemma 5. The line $\overline{X_{\tau_{i}}, X_{k(i)}}$, which is part of the convex hull, creates a positive drift out of the lens. Since the diameter of the lens is of order $d_{\tau_{i}}$ it will take the walker a time of order $d_{\tau_{i}}$ to reach the boundary of the lens. In fact we shall show, see Lemma 5, that it is exponentially costly for the walk to stay in the lens for a time longer than the order of $d_{\tau_{i}}$. This is enough to make sure that the walk is not slowed down too much by occasionally resisting the first mechanism and getting lost in the lens.

To make this idea precise we need to introduce more notation. The point

$$
Y_{i}:=\frac{X_{\tau_{i}}+X_{k(i)}}{2} \quad(i \geqslant 1)
$$

will serve as the "center" of $K_{\tau_{i}}$ and the "radius"

$$
R_{i, n}:=\left\|X_{\tau_{i}+n}-Y_{i}\right\| \quad(i \geqslant 1, n \geqslant 0)
$$

is the distance of $X_{\tau_{i}+n}$ from this center. The orthogonal projection of $X_{\tau_{i}+n}$ onto the straight line passing through $X_{\tau_{i}}$ and $X_{k(i)}$ will be called $Z_{i, n}(i \geqslant 1, n \geqslant 0)$. The distance of $X_{\tau_{i}+n}$ from this line is denoted by

$$
D_{i, n}:=\left\|X_{\tau_{i}+n}-Z_{i, n}\right\| \quad(i \geqslant 1, n \geqslant 0) .
$$

For the following definitions we assume $i, n \geqslant 1$ and $\tau_{i}+n<\tau_{i+1}$. In particular, due to (3), we assume that at time $\tau_{i}+n$ the walk has not yet left the lens created at time $\tau_{i}$. This implies that $Z_{i, n} \in \overline{X_{\tau_{i}}, X_{k(i)}}$ and that $X_{\tau_{i}}$ and 

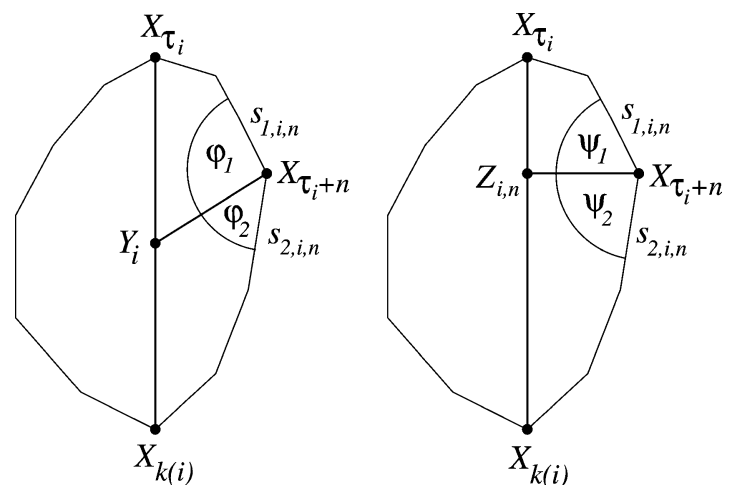

Fig. 4. General notation.

$X_{k(i)}$ are still boundary points of $K_{\tau_{i}+n}$, as shown in Figs. 2 and 4. Hence if we start in $X_{\tau_{i}+n}$ and follow the two boundary line segments emanating from $X_{\tau_{i}+n}$ we will eventually reach $X_{\tau_{i}}$ and $X_{k(i)}$. The boundary line segment whose continuation leads first to $K_{\tau_{i}}$ and then to $X_{k(i)}$ is called $s_{1, i, n}$, while the other line segment starting in $X_{\tau_{i}+n}$ is denoted by $s_{2, i, n}$, see Fig. 4 . The angle between $s_{j, i, n}$ and $\overline{X_{\tau_{i}+n}, Y_{i}}$ is called $\varphi_{j, i, n} \in[0, \pi](j=1,2)$, see the left part of Fig. 4. Similarly, the angle between $s_{j, i, n}$ and $\overline{X_{\tau_{i}+n}, Z_{i, n}}$ is denoted by $\psi_{j, i, n} \in[0, \pi](j=1,2)$, see the right part of Fig. 4. Occasionally, we will dropped the subscripts $i$ and $n$ from $\varphi$ and $\psi$. Since $K_{\tau_{i}+n}$ is convex,

$$
\varphi_{1}+\varphi_{2}=\psi_{1}+\psi_{2} \leqslant \pi
$$

Furthermore, $\left|\varphi_{1}-\psi_{1}\right|$ is one of the angles in a right angled triangle, namely the triangle with vertices $X_{\tau_{i}+n}, Y_{i}$ and $Z_{i, n}$. Hence,

$$
\left|\varphi_{1}-\psi_{1}\right|=\left|\varphi_{2}-\psi_{2}\right| \leqslant \pi / 2
$$

\section{Some sub- and supermartingales}

The following result shows that for every $i \geqslant 1$, both $\left(R_{i, n}\right)_{n}$ and $\left(D_{i, n}\right)_{n}\left(1 \leqslant n<\tau_{i+1}-\tau_{i}\right)$ are submartingales.

Lemma 2. For all $i, n \geqslant 1, P$-a.s. on $\left\{\tau_{i}+n<\tau_{i+1}\right\}$,

$$
\begin{aligned}
& E\left[R_{i, n+1}-R_{i, n} \mid \mathcal{F}_{\tau_{i}+n}\right] \geqslant \frac{\sin \varphi_{1, i, n}+\sin \varphi_{2, i, n}}{2 \pi} \geqslant \frac{\sin \varphi_{1, i, n}}{2 \pi} \geqslant 0, \\
& E\left[D_{i, n+1}-D_{i, n} \mid \mathcal{F}_{\tau_{i}+n}\right] \geqslant \frac{\sin \psi_{1, i, n}+\sin \psi_{2, i, n}}{2 \pi} \geqslant \frac{\sin \psi_{1, i, n}}{2 \pi} \geqslant 0
\end{aligned}
$$

and

$$
E\left[D_{i, n+1}-D_{i, n}+R_{i, n+1}-R_{i, n} \mid \mathcal{F}_{\tau_{i}+n}\right] \geqslant c_{2}
$$

for some constant $c_{2}>0$.

Fig. 5 shows examples in which the expected increments of $R_{i, n}$ and $D_{i, n}$ are close to 0 , thus explaining, why we are not able to bound in (6) and (7) these expected increments individually away from 0 . Note however, that in both situation depicted in Fig. 5, if the expected increment of $R_{i, n}$ or of $D_{i, n}$ is small then the expected increment of the other quantity is large. This confirms that the expected increments of $R_{i, n}$ and $D_{i, n}$ cannot both be small at the same time, see (8). 

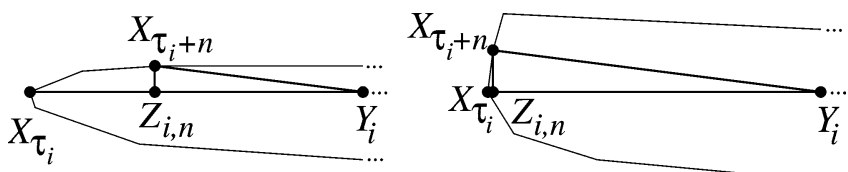

Fig. 5. The expected increment of $R_{i, n}$ is small in the left figure and large in the right figure. For $D_{i, n}$ it is the other way round.

Proof of Lemma 2. We fix $i, n \geqslant 1$ and drop them as subscripts of $\varphi_{j, i, n}$ and $\psi_{j, i, n}(j=1,2)$. Then the following statements hold on the event $\left\{\tau_{i}+n<\tau_{i+1}\right\}$. Consider the angle between $\overline{Y_{i}, X_{\tau_{i}+n}}$ and $\overline{X_{\tau_{i}+n}, X_{\tau_{i}+n+1}}$ which includes $s_{2, i, n}$. This angle is chosen uniformly at random from the interval $\left[\varphi_{2}, 2 \pi-\varphi_{1}\right]$. Hence we get by a change of basis argument using $\overline{Y_{i}, X_{\tau_{i}+n}}$ for the first basis vector,

$$
\begin{aligned}
E\left[R_{i, n+1} \mid \mathcal{F}_{\tau_{i}+n}\right] & =\frac{1}{\left(2 \pi-\varphi_{1}\right)-\varphi_{2}} \int_{\varphi_{2}}^{2 \pi-\varphi_{1}}\left\|\left(R_{i, n}, 0\right)-(\cos \varphi, \sin \varphi)\right\| \mathrm{d} \varphi \\
& \geqslant \frac{1}{2 \pi-\varphi_{1}-\varphi_{2}} \int_{\varphi_{2}}^{2 \pi-\varphi_{1}}\left|R_{i, n}-\cos \varphi\right| \mathrm{d} \varphi \\
& \geqslant R_{i, n}+\frac{1}{2 \pi-\varphi_{1}-\varphi_{2}} \int_{\varphi_{2}}^{2 \pi-\varphi_{1}}-\cos \varphi \mathrm{d} \varphi \\
& =R_{i, n}+\frac{\sin \varphi_{1}+\sin \varphi_{2}}{2 \pi-\varphi_{1}-\varphi_{2}} \geqslant R_{i, n}+\frac{\sin \varphi_{1}+\sin \varphi_{2}}{2 \pi},
\end{aligned}
$$

which shows (6). Similarly, (7) follows from

$$
E\left[D_{i, n+1} \mid \mathcal{F}_{\tau_{i}+n}\right]=\frac{1}{\left(2 \pi-\psi_{1}\right)-\psi_{2}} \int_{\psi_{2}}^{2 \pi-\psi_{1}}\left|D_{i, n}-\cos \psi\right| \mathrm{d} \psi .
$$

For the proof of (8) we assume without loss of generality $\varphi_{1} \leqslant \pi / 2$. Indeed, otherwise $\varphi_{2} \leqslant \pi / 2$ because of $\varphi_{1}+\varphi_{2} \leqslant \pi$, see (4), and in the following proof one only has to replace the subscript 1 by the subscript 2 and swap $X_{\tau_{i}}$ and $X_{k(i)}$. By (6) and (7),

$$
E\left[D_{i, n+1}-D_{i, n}+R_{i, n+1}-R_{i, n} \mid \mathcal{F}_{\tau_{i}+n}\right] \geqslant \frac{\sin \varphi_{1}+\sin \psi_{1}}{2 \pi} .
$$

We will show that the right side of (9) is always greater than $c_{2}:=\left(4 \pi^{2}\right)^{-1}$. Assume that it is less than $c_{2}$. Then

$$
\sin \varphi_{1}, \sin \psi_{1} \leqslant(2 \pi)^{-1}
$$

and hence $\varphi_{1} \leqslant(\pi / 2) \sin \varphi_{1} \leqslant 1 / 4$ by concavity of $\sin$ on $[0, \pi / 2]$. Similarly, (10) implies that either $\psi_{1} \leqslant 1 / 4$ or $\pi-\psi_{1} \leqslant 1 / 4$. Due to $\left|\varphi_{1}-\psi_{1}\right| \leqslant \pi / 2$, see (5), the latter case is impossible. Therefore,

$$
\left|\varphi_{1}-\psi_{1}\right| \leqslant \max \left\{\left|\varphi_{1}\right|,\left|\psi_{1}\right|\right\} \leqslant 1 / 4 \text {. }
$$

The angle $\alpha \in[0, \pi / 2]$ between $\overline{Z_{i, n}, X_{\tau_{i}+n}}$ and $\overline{X_{\tau_{i}+n}, X_{\tau_{i}}}$ is less than or equal to $\psi_{1}$. Consequently,

$$
\sin \psi_{1} \geqslant \sin \alpha=\frac{\left\|X_{\tau_{i}}-Z_{i, n}\right\|}{\left\|X_{\tau_{i}}-X_{\tau_{i}+n}\right\|} \geqslant \frac{\left\|X_{\tau_{i}}-Y_{i}\right\|-\left\|Y_{i}-Z_{i, n}\right\|}{d_{\tau_{i}}} .
$$


However, since $Y_{i}, X_{\tau_{i}+n} \in K_{\tau_{i}+n}$,

$$
R_{i, n}=\left\|Y_{i}-X_{\tau_{i}+n}\right\| \leqslant d_{\tau_{i}+n}=d_{\tau_{i}} .
$$

Therefore,

$$
\sin \psi_{1} \stackrel{(12)}{\geqslant} \frac{d_{\tau_{i}} / 2}{d_{\tau_{i}}}-\frac{\left\|Y_{i}-Z_{i, n}\right\|}{\left\|Y_{i}-X_{\tau_{i}+n}\right\|}=\frac{1}{2}-\sin \left|\varphi_{1}-\psi_{1}\right| \stackrel{(11)}{\geqslant} \frac{1}{2}-\frac{1}{4}=\frac{1}{4},
$$

which contradicts (10).

We fix the constants

$$
\beta:=1+4 \pi \sqrt{8}>30 \quad \text { and } \quad \gamma:=\frac{1}{2 \beta}<\frac{1}{60} .
$$

Whenever $\tau_{i}<\infty$ we denote the first exit time after $\tau_{i}$ from $B\left(X_{\tau_{i}}, \gamma d_{\tau_{i}}\right)$ by

$$
\gamma_{i+1}:=\inf \left\{n>\tau_{i}:\left\|X_{n}-X_{\tau_{i}}\right\|>\gamma d_{\tau_{i}}\right\} \quad(\leqslant \infty),
$$

see Fig. 2. If $i \geqslant 1$ and $n \geqslant 0$ then we shall call $n \operatorname{good}$ for $i$ if $n=0$ or if

$$
\tau_{i}+n<\tau_{i+1} \wedge \gamma_{i+1} \quad \text { and } \quad E\left[R_{i, n+1}-R_{i, n} \mid \mathcal{F}_{\tau_{i}+n}\right] \geqslant \frac{1}{\pi \sqrt{8}} \quad P \text {-a.s. }
$$

This means, $n \geqslant 1$ is good for $i$ if at time $\tau_{i}+n$ the walker has not yet left the intersection of the small ball around $X_{\tau_{i}}$ and the lens shown in Fig. 2 and, roughly speaking, feels a substantial centrifugal force pushing it away from the center $Y_{i}$. Good times help the walker to leave the lens shortly after $\tau_{i}$ and closely to the point $X_{\tau_{i}}$.

Next we introduce an Azuma type inequality for a certain family of supermartingales, which will help make this idea more precise.

Lemma 3. There are constants $c_{3}>0, c_{4}>0$ and $1 \leqslant c_{5}<\infty$ such that $P$-a.s. for all $i \geqslant 1, n \geqslant 0$,

$$
E\left[M_{i, n} \mid \mathcal{F}_{\tau_{i}}\right] \leqslant c_{5} \exp \left(-c_{4} n\right),
$$

where

$$
\begin{aligned}
& M_{i, n}:=\mathbf{1}\left\{\tau_{i}+n<\tau_{i+1}\right\} \exp \left(c_{3} \sum_{m=1}^{n} W_{i, m}\right) \text { and } \\
& W_{i, m}:=D_{i, m-1}-D_{i, m}+\beta\left(R_{i, m-1}-R_{i, m}\right)+4 \mathbf{1}\{m-1 \text { is good for } i\} .
\end{aligned}
$$

Proof. Fix $i \geqslant 1$. Firstly, we shall prove that for suitable $c_{4}>0$,

$$
E\left[M_{i, n+1} \mid \mathcal{F}_{\tau_{i}+n}\right] \leqslant \exp \left(-c_{4}\right) M_{i, n} \quad P \text {-a.s. for all } n \geqslant 1,
$$

thus showing that $\left(M_{i, n}\right)_{n \geqslant 1}$ is an exponentially fast decreasing submartingale. Since $W_{i, m}$ is $\mathcal{F}_{\tau_{i}+m}$-measurable $(m \geqslant 1)$, we have

$$
\begin{aligned}
E\left[M_{i, n+1} \mid \mathcal{F}_{\tau_{i}+n}\right] & =M_{i, n} E\left[\exp \left(c_{3} W_{i, n+1}\right), \tau_{i}+n+1<\tau_{i+1} \mid \mathcal{F}_{\tau_{i}+n}\right] \\
& \leqslant M_{i, n} E\left[\exp \left(c_{3} W_{i, n+1}\right) \mid \mathcal{F}_{\tau_{i}+n}\right] .
\end{aligned}
$$

Therefore is suffices to show for the proof of (16) that on the event $\left\{\tau_{i}+n<\tau_{i+1}\right\}, E\left[\exp \left(c_{3} W_{i, n+1}\right) \mid \mathcal{F}_{\tau_{i}+n}\right]<1$ for some suitable $c_{3}>0$. However, since the $W_{i, m}$ 's $(m \geqslant 1)$ are uniformly bounded by a constant this follows from the fact that on the event $\left\{\tau_{i}+n<\tau_{i+1}\right\}$, 


$$
\begin{aligned}
E\left[W_{i, n+1} \mid \mathcal{F}_{\tau_{i}+n}\right]= & E\left[D_{i, n}-D_{i, n+1}+R_{i, n}-R_{i, n+1} \mid \mathcal{F}_{\tau_{i}+n}\right] \\
& +4\left(\pi \sqrt{8} E\left[R_{i, n}-R_{i, n+1} \mid \mathcal{F}_{\tau_{i}+n}\right]+\mathbf{1}\{n \text { is good for } i\}\right) \\
\leqslant & -c_{2}<0
\end{aligned}
$$

$P$-a.s. by virtue of definition (15) and Lemma 2 (6), (8). Using (16) for induction over $n$ we obtain by the tower property,

$$
E\left[M_{i, n} \mid \mathcal{F}_{\tau_{i}}\right] \leqslant \exp \left(-c_{4}(n-1)\right) E\left[M_{i, 1} \mid \mathcal{F}_{\tau_{i}}\right] \quad P \text {-a.s. }
$$

for all $n \geqslant 1$. Since $M_{i, 1}$ is bounded by a constant this finishes the proof.

\section{Exponential moments of $\tau_{i+1}-\tau_{i}$}

The next two lemmas provide tools needed to bound in Proposition 6 the times $\Delta_{i}$ during which the diameter does not increase. The first lemma shows that the walk cannot spend too much time inside the lens and the small ball around $X_{\tau_{i}}$ shown in Fig. 2.

Lemma 4. For all $i \geqslant 1, n \geqslant 0, P$-a.s.

$$
P\left[\tau_{i}+n<\tau_{i+1} \wedge \gamma_{i+1} \mid \mathcal{F}_{\tau_{i}}\right] \leqslant c_{5} \exp \left(-c_{4} n\right),
$$

where $c_{4}$ and $c_{5}$ are as in Lemma 3.

The second lemma gives a first crude upper bound for $\Delta_{i}$.

Lemma 5. There is a finite constant $c_{6}$ such that for all $i \geqslant 1, n \geqslant 0, P$-a.s.

$$
P\left[\Delta_{i}>n \mid \mathcal{F}_{\tau_{i}}\right] \leqslant c_{5} \exp \left(c_{4}\left(c_{6} d_{\tau_{i}}-n\right)\right),
$$

where $c_{4}$ and $c_{5}$ are as in Lemma 3.

In the proof of both lemmas we will use the fact that the sum in the definition of $M_{i, n}$ is in part telescopic, i.e.

$$
\sum_{m=1}^{n} W_{i, m}=-D_{i, n}+\beta\left(R_{i, 0}-R_{i, n}\right)+4 \sum_{m=1}^{n} \mathbf{1}\{m-1 \text { is good for } i\}
$$

since $D_{i, 0}=0$.

Proof of Lemma 4. For $n=0$ the statement is true since $c_{5} \geqslant 1$. Fix $n \geqslant 1$. The statement follows from Lemma 3 and (17) once we have shown that on the event $\left\{\tau_{i}+n<\tau_{i+1} \wedge \gamma_{i+1}\right\}$, the right-hand side of (17) is nonnegative. First we will show that on $\left\{\tau_{i}+n<\tau_{i+1} \wedge \gamma_{i+1}\right\}$,

$$
D_{i, n}+\beta\left(R_{i, n}-R_{i, 0}\right) \leqslant 2\left(D_{i, n}-\left\|X_{\tau_{i}}-Z_{i, n}\right\|\right) .
$$

This is done by brute force. For abbreviation we set $d:=d_{\tau_{i}}, y:=D_{i, n}$ and $x:=\left\|X_{\tau_{i}}-Z_{i, n}\right\|$ and note that on $\left\{\tau_{i}+n<\tau_{i+1} \wedge \gamma_{i+1}\right\}$ we have $x, y \in[0, \gamma d]$. Observe that $x$ and $y$ play the role of Cartesian coordinates of $X_{\tau_{i}+n}$, see Fig. 6. Using $R_{i, 0}=d / 2$ and $R_{i, n}=\sqrt{(d / 2-x)^{2}+y^{2}}$ we see that (18) is equivalent to

$$
\beta \sqrt{(d / 2-x)^{2}+y^{2}} \leqslant y-2 x+\beta d / 2 .
$$

Both sides of this inequality are nonnegative since $x$ is less than $\gamma d$, which is tiny compared to $\beta d$. Taking the square and rearranging shows that (18) is equivalent to

$$
x\left(4 x-4 y-2 \beta d-\beta^{2} x+\beta^{2} d\right)+y\left(y+\beta d-\beta^{2} y\right) \geqslant 0 .
$$




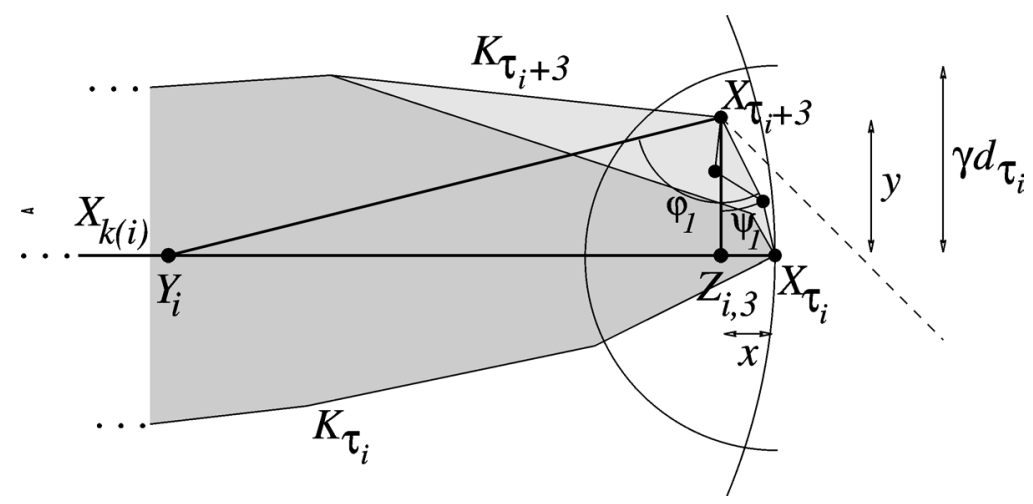

Fig. 6. More realistic and detailed than Fig. 3. The darkly shaded convex hull $K_{\tau_{i}}$ at time $\tau_{i}$ has been enlarged after three steps by the lightly shaded part. $j=3$ is good for $i$ since it satisfies the sufficient criterion $\psi_{1, i, 3} \leqslant \pi / 4$, see (22), which corresponds to the fact that the dashed line, which intersects the horizontal axis at an angle of $\pi / 4$, does not intersect $K_{\tau_{i}+3}^{o} \cdot j=1$ is also good for $i$ for the same reason, while $j=2$ might be good for $i$ but fails to satisfy the sufficient condition (22), since the corresponding dashed line starting in $X_{\tau_{i}+2}$ would have intersected $K_{\tau_{i}+2}^{o}$.

Since $x, y \in[0, \gamma d]$ and $\beta \gamma=1 / 2$, see (14), the terms $\beta^{2} d$ in the first bracket and $\beta d$ in the second bracket are the dominant terms, respectively, which shows that (19) and thus (18) holds. For the proof that the right-hand side of (17) is indeed nonnegative on $\left\{\tau_{i}+n<\tau_{i+1} \wedge \gamma_{i+1}\right\}$ it therefore suffices to show that,

$$
D_{i, n}-\left\|X_{\tau_{i}}-Z_{i, n}\right\| \leqslant 2 \sum_{j=0}^{n-1} \mathbf{1}\{j \text { is good for } i\} .
$$

Both $D_{i, j}$ and $-\left\|X_{\tau_{i}}-Z_{i, j}\right\|$ can increase by at most 1 if $j$ increases by 1 . Therefore, the left-hand side of (20) is less than or equal to $2\left(\# J_{i, n}\right)$ where

$$
J_{i, n}:=\left\{0 \leqslant j<n \mid \forall 0 \leqslant m<j: D_{i, m}-\left\|X_{\tau_{i}}-Z_{i, m}\right\| \leqslant D_{i, j}-\left\|X_{\tau_{i}}-Z_{i, j}\right\|\right\} .
$$

Hence it suffices to show that the elements of $J_{i, n}$ are good for $i$. Note that $j=0 \in J_{i, n}$ is good for $i$ by definition of being good. So fix $1 \leqslant j \in J_{i, n}$. By Lemma 2 (6) it is enough to show that $\sin \varphi_{1, i, j} \geqslant 2^{-1 / 2}$, that is

$$
\varphi_{1, i, j} \in[\pi / 4,3 \pi / 4] \text {. }
$$

On the one hand, $\varphi_{1}-\psi_{1}$ is close to $\pi / 2$, as can be seen in Fig. 6. More precisely,

$$
\begin{aligned}
\sin \left(\varphi_{1}-\psi_{1}\right) & =\frac{\left\|Y_{i}-Z_{i, j}\right\|}{\left\|Y_{i}-X_{\tau_{i}+j}\right\|} \geqslant \frac{\left\|Y_{i}-X_{\tau_{i}}\right\|-\left\|X_{\tau_{i}}-Z_{i, j}\right\|}{\left\|Y_{i}-X_{\tau_{i}}\right\|+\left\|X_{\tau_{i}}-X_{\tau_{i}+j}\right\|} \\
& \geqslant \frac{d_{\tau_{i}} / 2-\gamma d_{\tau_{i}}}{d_{\tau_{i}} / 2+\gamma d_{\tau_{i}}}=\frac{1-2 \gamma}{1+2 \gamma} \geqslant \frac{1}{\sqrt{2}}=\sin \frac{\pi}{4} .
\end{aligned}
$$

Since $0 \leqslant \psi_{1} \leqslant \varphi_{1}$ this implies $\varphi_{1} \geqslant \pi / 4$, thus proving the first part of (21). On the other hand, $\varphi_{1}-\psi_{1} \leqslant \pi / 2$, see (5). Hence all that remains to be shown for the completion of the proof of (21) is that

$$
\psi_{1, i, j} \leqslant \pi / 4 \text {. }
$$

Consider the half line (dashed in Fig. 6) starting at $X_{\tau_{i}+j}$ which includes an angle of $\pi / 4$ with $\overline{X_{\tau_{i}+j}, Z_{i, j}}$ that contains $s_{1, i, j}$. We claim that this line does not intersect $K_{\tau_{i}+j}^{o}$. This would imply (22). To prove this claim observe that for any $c>0$ the set of possible values for $X_{\tau_{i}+m}$ with $D_{i, m}-\left\|X_{\tau_{i}}-Z_{i, m}\right\|=c$ is a line parallel to the half line just described. Since $j \in J_{i, n}$ the walker did not cross between time $\tau_{i}$ and time $\tau_{i}+j-1$ the dashed line passing through $X_{\tau_{i}+j}$. Consequently, it suffices to show that the dashed line does not intersect $K_{\tau_{i}}^{o}$. If it did intersect $K_{\tau_{i}}^{o}$ 
then this would force the walker on its way from $X_{\tau_{i}}$ to $X_{\tau_{i}+j}$ to cross the dashed line strictly before time $\tau_{i}+j$, which is impossible as we just saw.

Proof of Lemma 5. Again, the statement is true for $n=0$ since $c_{5} \geqslant 1$. For $n \geqslant 1$, by the Pythagorean theorem and (13), $D_{i, n} \leqslant R_{i, n} \leqslant d_{\tau_{i}}$ on the event $\left\{\tau_{i}+n<\tau_{i+1}\right\}$. Thus on this event the right-hand side of (17) is greater than or equal to $-d_{\tau_{i}}+\beta\left(0-d_{\tau_{i}}\right)+0$. Hence due to Lemma 3 and (17), $P$-a.s. for all $n \geqslant 1$,

$$
\exp \left(-c_{3}(1+\beta) d_{\tau_{i}}\right) P\left[\tau_{i}+n<\tau_{i+1} \mid \mathcal{F}_{\tau_{i}}\right] \leqslant c_{5} \exp \left(-c_{4} n\right),
$$

which is equivalent to the claim of the lemma with $c_{6}:=c_{3}(1+\beta) / c_{4}$.

The following result is stronger than Lemma 5.

Proposition 6. $\tau_{i}<\infty P$-a.s. for all $i \geqslant 0$. Moreover, there are positive constants $c_{7}, c_{8}$ and finite constants $c_{9}$ and $c_{10} \geqslant 1$ such that for all $i \geqslant 0$ and $n \geqslant 0, P$-a.s.,

$$
\begin{aligned}
& P\left[\Delta_{i} \geqslant n \mid \mathcal{F}_{\tau_{i}}\right] \leqslant c_{9} \exp \left(-c_{7} n\right) \quad \text { and } \\
& E\left[\exp \left(c_{8} \Delta_{i}\right) \mid \mathcal{F}_{\tau_{i}}\right] \leqslant c_{10} .
\end{aligned}
$$

Proof. (24) is an immediate consequence of (23). For the proof of (23) choose

$$
c_{7}:=\frac{\gamma c_{4}}{c_{6}+\gamma} \quad \text { and } \quad c_{9}:=c_{5} \mathrm{e}^{c_{4}}
$$

with $c_{4}, c_{5}$ and $c_{6}$ according to Lemma 5. We only need to show that (23) holds for all $i \geqslant 1$ with $\tau_{i}<\infty$. Indeed, the case $i=0$ is trivial since $\tau_{0}=0, \tau_{1}=1$ and hence $\Delta_{0}=1$. Moreover, since (23) implies $\Delta_{i}<\infty P$-a.s., we then have $\tau_{i}=\Delta_{0}+\cdots+\Delta_{i-1}<\infty$ as well.

Fix $i \geqslant 1$ and $n \geqslant 0$. We distinguish three cases:

$$
\left\{n<\gamma d_{\tau_{i}}\right\}, \quad\left\{\gamma d_{\tau_{i}} \leqslant n<\left(c_{6}+\gamma\right) d_{\tau_{i}}\right\} \quad \text { and } \quad\left\{\left(c_{6}+\gamma\right) d_{\tau_{i}} \leqslant n\right\} .
$$

Note that these events are elements of $\mathcal{F}_{\tau_{i}}$ and partition $\Omega$. By definition of $\gamma_{i+1}$,

$$
\gamma_{i+1} \geqslant \tau_{i}+\left\lceil\gamma d_{\tau_{i}}\right\rceil
$$

since the walker takes steps of length one. Therefore, on $\left\{n<\gamma d_{\tau_{i}}\right\}$,

$$
\begin{aligned}
& P\left[\Delta_{i}>n \mid \mathcal{F}_{\tau_{i}}\right] \stackrel{(25)}{=} P\left[\tau_{i}+n<\tau_{i+1} \wedge \gamma_{i+1} \mid \mathcal{F}_{\tau_{i}}\right] \\
& \stackrel{\text { Lemma } 4}{\leqslant} c_{5} \exp \left(-c_{4} n\right) \stackrel{(25)}{\leqslant} c_{9} \exp \left(-c_{7} n\right) .
\end{aligned}
$$

On $\left\{\gamma d_{\tau_{i}} \leqslant n<\left(c_{6}+\gamma\right) d_{\tau_{i}}\right\}$,

$$
\begin{aligned}
& P\left[\Delta_{i}>n \mid \mathcal{F}_{\tau_{i}}\right] \leqslant P\left[\tau_{i}+\left\lceil\gamma d_{\tau_{i}}\right\rceil<\tau_{i+1} \mid \mathcal{F}_{\tau_{i}}\right] \stackrel{(26)}{\leqslant} P\left[\tau_{i}+\left\lceil\gamma d_{\tau_{i}}\right\rceil-1<\tau_{i+1} \wedge \gamma_{i+1} \mid \mathcal{F}_{\tau_{i}}\right] \\
& \stackrel{\text { Lemma }}{\leqslant} c_{5} \exp \left(-c_{4}\left(\gamma\left\lceil d_{\tau_{i}}\right\rceil-1\right)\right) \stackrel{(25)}{\leqslant} c_{9} \exp \left(-c_{7} n\right) .
\end{aligned}
$$

Finally, on $\left\{\left(c_{6}+\gamma\right) d_{\tau_{i}} \leqslant n\right\}$,

$$
P\left[\Delta_{i}>n \mid \mathcal{F}_{\tau_{i}}\right] \stackrel{\text { Lemma } 5}{\leqslant} c_{5} \exp \left(c_{4}\left(c_{6} d_{\tau_{i}}-n\right)\right) \stackrel{(25)}{\leqslant} c_{9} \exp \left(-c_{7} n\right),
$$

where the last inequality can easily be checked. 


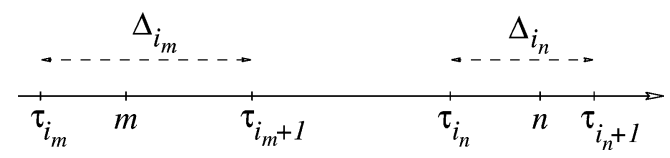

Fig. 7.

\section{Linear growth of the diameter and proof of Theorem 1}

The following result (with $m=0$ ) implies that $\left(d_{n}\right)_{n}$ has a positive lim inf speed.

Lemma 7. There are constants $c_{11}>0$ and $c_{12}<\infty$ such that for all $0 \leqslant m \leqslant n$,

$$
E\left[\exp \left(d_{m}-d_{n}\right)\right] \leqslant c_{12}(n+1) \exp \left(c_{11}(m-n)\right) .
$$

For the proof of this lemma and of Theorem 1 we need the following definition: Given $n \geqslant 0$ let $i_{n}:=\sup \left\{i \geqslant 0 \mid \tau_{i} \leqslant n\right\}$. Note that

$$
d_{\tau_{i_{n}}}=d_{n} \quad \text { and } \quad i_{n} \leqslant \tau_{i_{n}} \leqslant n<\tau_{i_{n+1}} .
$$

Proof of Lemma 7. The case $n=m$ is trivial. So let $0 \leqslant m<n$ and set

$$
f(m, n):=\frac{n-m}{2 n}>0 \quad \text { and } \quad g(m, n):=\frac{c_{8} f(m, n)}{2 \ln c_{10}}>0,
$$

where $c_{8}$ and $c_{10}$ are according to Proposition 6. A simple union bound yields

$$
\begin{aligned}
E\left[\mathrm{e}^{d_{m}-d_{n}}\right] \leqslant \mathrm{I}+\mathrm{II}+\mathrm{III}, \quad \text { where } \\
\mathrm{I}:=P\left[\tau_{i_{m}+1}-m \geqslant f(m, n) n\right], \\
\mathrm{II}:=P\left[\tau_{i_{m}+1}-m<f(m, n) n, i_{n}<i_{m}+\lceil g(m, n) n\rceil\right] \text { and } \\
\mathrm{III}:=E\left[\exp \left(d_{m}-d_{n}\right), i_{n} \geqslant i_{m}+\lceil g(m, n) n\rceil\right],
\end{aligned}
$$

see also Fig. 7. Here term I corresponds to the situation in which after time $m$ the diameter does not increase for an untypical long while. Term II handles the case in which the diameter does increase shortly after time $m$, as it should, but not often enough in the remaining time until $n$. The third term III considers the original random variable on the typical event that the number of times at which the diameter increases is at least proportional to $n$ with a constant of proportionality not too small.

It suffices to show that each of these three terms decays as $n \rightarrow \infty$ in the way claimed in Lemma 7 with constants $c_{11}$ and $c_{12}$ independent of $n$ and $m$. As for the first term,

$$
\begin{aligned}
& \mathrm{I} \leqslant P\left[\Delta_{i_{m}} \geqslant\lceil f(m, n) n\rceil\right] \stackrel{(27)}{=} \sum_{i=0}^{m} P\left[i_{m}=i, \Delta_{i} \geqslant\lceil f(m, n) n\rceil\right] \\
& \stackrel{(23)}{\leqslant} c_{9}(m+1) \mathrm{e}^{-c_{7}\lceil f(m, n) n\rceil} \leqslant c_{9}(n+1) \mathrm{e}^{c_{7}(m-n) / 2},
\end{aligned}
$$

which is an upper bound like the one requested in Lemma 7. The second term is estimated as follows.

$$
\begin{aligned}
& \mathrm{II} \stackrel{(27)}{=} P\left[\tau_{i_{m}+1}<m+f(m, n) n, n<\tau_{i_{n}+1} \leqslant \tau_{i_{m}+\lceil g(m, n) n\rceil}\right] \\
& \quad \stackrel{(28)}{=} P\left[\tau_{i_{m}+\lceil g(m, n) n\rceil}-\tau_{i_{m}+1} \geqslant n-m-f(m, n) n=f(m, n) n\right] \\
& \quad \leqslant E\left[\exp \left(c_{8}\left(\tau_{i_{m}+\lceil g(m, n) n\rceil}-\tau_{i_{m}+1}-f(m, n) n\right)\right)\right]
\end{aligned}
$$




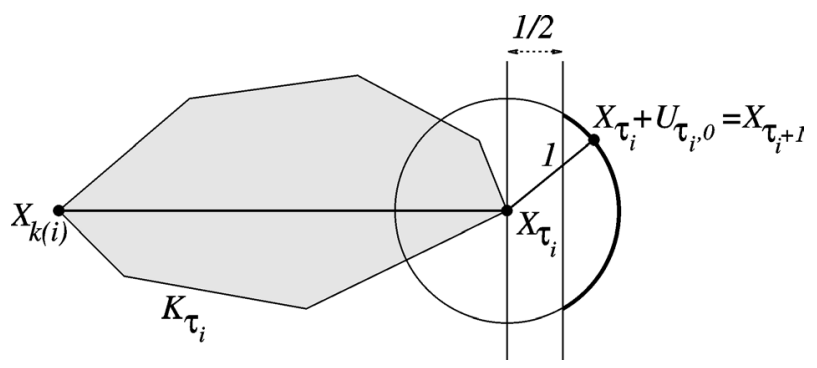

Fig. 8. The event $A_{i}$ occurs if the first trial point sampled lies on the bold arc.

$$
\begin{aligned}
& =\mathrm{e}^{-c_{8} f(m, n) n} \sum_{k \geqslant 1} E\left[\exp \left(c_{8}\left(\tau_{k+\lceil g(m, n) n\rceil-1}-\tau_{k}\right)\right), i_{m}+1=k\right] \\
& =\mathrm{e}^{-c_{8} f(m, n) n} \sum_{k \geqslant 1} E\left[\prod_{i=0}^{\lceil g(m, n) n\rceil-2} \exp \left(c_{8} \Delta_{k+i}\right), i_{m}+1=k\right] .
\end{aligned}
$$

Note that $\left\{i_{m}+1=k\right\}$ is the event that $\tau_{k}$ is the first time after time $m$ at which the diameter increases. Therefore,

$$
\left\{i_{m}+1=k\right\} \in \mathcal{F}_{\tau_{k}} .
$$

Moreover, the increments $\Delta_{k+i}$ are measurable with respect to $\mathcal{F}_{\tau_{k+i+1}}$. Consequently, by conditioning in (29) on $\mathcal{F}_{\tau_{k+\lceil g(m, n) n\rceil-2}}$ and applying Proposition 6 (24) with $i=k+\lceil g(m, n) n\rceil-2$ we conclude

$$
\mathrm{II} \leqslant \mathrm{e}^{-c_{8} f(m, n) n} c_{10} \sum_{k \geqslant 1} E\left[\prod_{i=0}^{\lceil g(m, n) n\rceil-3} \exp \left(c_{8}\left(\Delta_{k+i}\right)\right), i_{m}+1=k\right] .
$$

Continuing in this way we obtain by induction after $\lceil g(m, n) n\rceil-1$ steps,

$$
\mathrm{II} \leqslant \mathrm{e}^{-c_{8} f(m, n) n} c_{10}^{\lceil g(m, n) n\rceil-1} \leqslant \mathrm{e}^{-c_{8} f(m, n) n} c_{10}^{g(m, n) n} \stackrel{(28)}{=} \mathrm{e}^{\left(c_{8} / 4\right)(m-n)},
$$

which is again of the form required in Lemma 7.

In order to demonstrate that also the third term III behaves properly we will show that the increments $d_{\tau_{i+1}}-d_{\tau_{i}}$, $i \geqslant 1$, have a uniformly positive chance of being larger than a fixed constant, say $1 / 2$, independently of the past. To this end, we may assume that the process $\left(X_{n}\right)_{n}$ is generated in the following way: There are i.i.d. random variables $U_{n, k}, n \geqslant 0, k \geqslant 0$, uniformly distributed on the unit circle centered in 0 such that $X_{n+1}=X_{n}+U_{n, k}$, where $k$ is the smallest integer such that $\overline{X_{n}, X_{n}+U_{n, k}}$ does not intersect $K_{n}$. Then for any $i \geqslant 1$, by definition of $\tau_{i}$,

$$
\left\{d_{\tau_{i+1}} \geqslant d_{\tau_{i}}+1 / 2\right\} \supseteq\left\{d_{\tau_{i}+1} \geqslant d_{\tau_{i}}+1 / 2\right\} \supseteq\left\{U_{\tau_{i}, 0} \cdot \frac{X_{\tau_{i}}-X_{k(i)}}{d_{\tau_{i}}} \geqslant \frac{1}{2}\right\}=: A_{i} .
$$

The reason for the second inclusion in (31) is illustrated in Fig. 8: If $A_{i}$ occurs then $K_{\tau_{i}}$ and $X_{\tau_{i}}+U_{\tau_{i}, 0}$ are separated by a slab of width $1 / 2$. In particular, the line connecting $X_{\tau_{i}}$ and $X_{\tau_{i}}+U_{\tau_{i}, 0}$ does not intersect $K_{\tau_{i}}$. Hence $\tau_{i+1}=\tau_{i}+1$ and $X_{\tau_{i+1}}=X_{\tau_{i}+1}=X_{\tau_{i}}+U_{\tau_{i}, 0}$. Therefore,

$$
d_{\tau_{i}+1} \geqslant\left\|X_{\tau_{i}+1}-X_{k(i)}\right\| \geqslant\left\|X_{\tau_{i}}-X_{k(i)}\right\|+1 / 2=d_{\tau_{i}}+1 / 2 .
$$

It follows from (31) that for all $1 \leqslant j_{1} \leqslant j_{2}$,

$$
d_{\tau_{j_{2}}}-d_{\tau_{j_{1}}} \geqslant \frac{1}{2} \sum_{i=j_{1}}^{j_{2}-1} \mathbf{1}\left\{d_{\tau_{i+1}} \geqslant d_{\tau_{i}}+1 / 2\right\} \geqslant \frac{1}{2} \sum_{i=j_{1}}^{j_{2}-1} \mathbf{1}\left\{A_{i}\right\}
$$


This estimate will be useful since the random variables

$$
\mathbf{1}\left\{A_{i}\right\}(i \geqslant 1) \text { are i.i.d. with } P\left[A_{i}\right]>0 .
$$

Indeed, let $\widetilde{\mathcal{F}}_{n}(n \geqslant 0)$ be the $\sigma$-field generated by $U_{m, k}, 0 \leqslant m<n, 0 \leqslant k$. Because of $\mathcal{F}_{n} \subseteq \widetilde{\mathcal{F}}_{n}$ we have $A_{j} \in \widetilde{\mathcal{F}}_{\tau_{i}}$ for all $1 \leqslant j<i$. Moreover, since the uniform distribution on the unit circle is invariant under rotations,

$$
A_{i} \text { is independent of } \widetilde{\mathcal{F}}_{\tau_{i}}(i \geqslant 1)
$$

and $P\left[A_{i} \mid \widetilde{\mathcal{F}}_{\tau_{i}}\right]=P\left[A_{i}\right]$ is just the length of the bold circle segment shown in Fig. 8 divided by $2 \pi$. This implies (33). Now we estimate III by

$$
\begin{aligned}
\mathrm{III} & \stackrel{(27)}{=} E\left[\exp \left(d_{\tau_{i_{m}}}-d_{\tau_{i_{n}}}\right), i_{n} \geqslant i_{m}+\lceil g(m, n) n\rceil\right] \\
& \leqslant E\left[\exp \left(d_{\tau_{i_{m}+1}}-d_{\tau_{i_{n}}}\right), i_{n} \geqslant\left(i_{m}+1\right)+\lceil g(m, n) n\rceil-1\right] \\
& \leqslant \sum_{k \geqslant 1} E\left[\exp \left(d_{\tau_{k}}-d_{\tau_{k+\lceil g(m, n) n\rceil-1}}\right), i_{m}+1=k\right] \\
& \stackrel{(32)}{\leqslant} \sum_{k \geqslant 1} E\left[\exp \left(-\frac{1}{2} \sum_{i=k}^{k+\lceil g(m, n) n\rceil-2} \mathbf{1}\left\{A_{i}\right\}\right), i_{m}+1=k\right] .
\end{aligned}
$$

As seen in (30), $\left\{i_{m}+1=k\right\} \in \mathcal{F}_{\tau_{k}} \subseteq \widetilde{\mathcal{F}}_{\tau_{k}}$. Therefore, after conditioning in (35) on $\mathcal{F}_{\tau_{k}}$, we see with the help of (34) for $i \geqslant k$ and (33) that the right-hand side of (35) equals

$$
E\left[\exp \left(-\frac{1}{2} A_{1}\right)\right]^{\lceil g(m, n) n\rceil-1}
$$

which decays as required in Lemma 7, see (28).

Lemma 7 directly implies a weaker version of Theorem 1 in which $\left\|X_{n}\right\|$ is replaced by $d_{n}$. For the full statement we need the following additional argument.

Proof of Theorem 1. (2) follows from (1) by the Borel-Cantelli lemma. For the proof of (1) pick $c_{11}$ and $c_{12}$ according to Lemma 7 and choose $c_{13}>0$ and $c_{1}>0$ small enough such that

$$
2 c_{13}-c_{11}<0 \text { and } 2 c_{1}-c_{11}\left(c_{13}-c_{1}\right)<0 \text {. }
$$

We denote by $M_{n}:=\max \left\{\left\|X_{m}\right\| \mid m \leqslant n\right\}$ the walker's maximal distance from the origin by time $n$. Note that $M_{n}$ and $d_{n}$ are related via

$$
M_{n} \leqslant d_{n} \leqslant 2 M_{n} \text { for all } n \geqslant 0
$$

because of $X_{0}=0$. By a union bound for any $n \geqslant 0$,

$$
\begin{aligned}
& P\left[\left\|X_{n}\right\| \leqslant c_{1} n\right] \leqslant P\left[\Delta_{i_{n}} \geqslant c_{1} n\right]+P\left[M_{n} \leqslant c_{13} n\right]+P\left[B_{n}\right], \quad \text { where } \\
& B_{n}:=\left\{\Delta_{i_{n}}<c_{1} n, M_{n}>c_{13} n,\left\|X_{n}\right\| \leqslant c_{1} n\right\} .
\end{aligned}
$$

It suffices to show that each one of the three terms on the right-hand side of (38) decays exponentially fast in $n$. As for the first term,

$$
P\left[\Delta_{i_{n}} \geqslant c_{1} n\right] \stackrel{(27)}{=} \sum_{i=0}^{n} P\left[i_{n}=i, \Delta_{i} \geqslant\left\lceil c_{1} n\right\rceil\right] \stackrel{(23)}{\leqslant} c_{9}(n+1) \mathrm{e}^{-c_{7} c_{1} n},
$$

which decays exponentially fast in $n$ indeed. So does the second term in (38) since by Chebyshev's inequality,

$$
P\left[M_{n} \leqslant c_{13} n\right] \stackrel{(37)}{\leqslant} P\left[d_{n} \leqslant 2 c_{13} n\right] \leqslant \mathrm{e}^{2 c_{13} n} E\left[\mathrm{e}^{-d_{n}}\right] \stackrel{\text { Lemma } 7}{\leqslant} c_{12}(n+1) \mathrm{e}^{\left(2 c_{13}-c_{11}\right) n},
$$


which decays exponentially fast due to the choice of $c_{13}$ in (36). Finally, we are going to bound the third term in (38), $P\left[B_{n}\right]$. Define the ladder times $\left(\mu_{j}\right)_{j}$ of the process $\left(M_{n}\right)_{n \geqslant 0}$ recursively by

$$
\mu_{0}:=0 \quad \text { and } \quad \mu_{j+1}:=\inf \left\{n>\mu_{j} \mid M_{n}>M_{\mu_{j}}\right\}
$$

In analogy to $\left(i_{n}\right)_{n}$ for $\left(\tau_{i}\right)_{i}$ we define for $\left(\mu_{j}\right)_{j}$ the increasing sequence $\left(j_{n}\right)_{n}$ by $j_{n}:=\sup \left\{j \geqslant 0 \mid \mu_{j} \leqslant n\right\}$ and note that $\mu_{j_{n}} \leqslant n<\mu_{j_{n}+1}$ and $M_{n}=\left\|X_{\mu_{j_{n}}}\right\|$. Hence on the event $B_{n}$,

$$
\left\|X_{\mu_{j_{n}}}-X_{n}\right\| \geqslant\left\|X_{\mu_{j_{n}}}\right\|-\left\|X_{n}\right\|=M_{n}-\left\|X_{n}\right\| \geqslant\left(c_{13}-c_{1}\right) n .
$$

Since the walker takes steps of length one, this implies $n-\mu_{j_{n}} \geqslant\left(c_{13}-c_{1}\right) n$ and therefore, on the event $B_{n}$,

$$
\mu_{j_{n}} \leqslant\left\lfloor\left(1-c_{13}+c_{1}\right) n\right\rfloor .
$$

On the other hand, on $B_{n}$,

$$
d_{n} \stackrel{(27)}{=} d_{\tau_{i_{n}}}=\left\|X_{\tau_{i_{n}}}-X_{k\left(i_{n}\right)}\right\| \leqslant\left\|X_{n}\right\|+\left\|X_{n}-X_{\tau_{i_{n}}}\right\|+\left\|X_{k\left(i_{n}\right)}\right\| \leqslant c_{1} n+\Delta_{i_{n}}+M_{n},
$$

where we used again the fact that the steps have length one to estimate the second term and $k\left(i_{n}\right) \leqslant i_{n} \leqslant n$ to bound the third term. Therefore the right-most term in (40) can be estimated on $B_{n}$ from above by

$$
c_{1} n+c_{1} n+M_{\mu_{j_{n}}} \stackrel{(37)}{\leqslant} 2 c_{1} n+d_{\mu_{j_{n}}} \stackrel{(39)}{\leqslant} 2 c_{1} n+d_{\left\lfloor\left(1-c_{13}+c_{1}\right) n\right\rfloor} .
$$

Consequently, by Chebyshev's inequality and Lemma 7 ,

$$
P\left[B_{n}\right] \leqslant P\left[d_{n}-d_{\left\lfloor\left(1-c_{13}+c_{1}\right) n\right\rfloor} \leqslant 2 c_{1} n\right] \leqslant c_{12}(n+1) \mathrm{e}^{\left(2 c_{1}-c_{11}\left(c_{13}-c_{1}\right)\right) n},
$$

which decays exponentially in $n$ due to the choice of $c_{13}$ and $c_{1}$ in (36).

\section{References}

[1] O. Angel, I. Benjamini, B. Virág, Random walks that avoid their past convex hull, Electron Comm. Probab. 8 (2003) 6-16.

[2] I. Benjamini, D.B. Wilson, Excited random walk, Electron Comm. Probab. 8 (2003) 86-92. 